\title{
EP-192
}

\section{Positive intraoperative pancreatic parenchymal resection margin: Is it a true indication of completion total pancreatectomy for pancreatic ductal adenocarcinoma?}

\author{
Ji Hye JUNG, Sokyung YOON, So Jeong YOON, Sang Hyun SHIN, Jin Seok HEO, In Woong HAN*
}

Division of Hepatobiliary-Pancreatic Surgery, Samsung Medical Center, Sungkyunkwan University School of Medicine, Seoul, Korea

Introduction: Total pancreatectomy (TP) has being performed to achieve radicality. In the cases that negative margin (R0) are achieved by performing intraoperative completion TP (cTP), we aimed to identify if they showed better outcomes compared to the cases that negative margin were not obtained despite CTP and the cases that R0 were confirmed after pancreaticoduodenectomy (PD).

Methods: 718 pancreatic ductal adenocarcinoma (PDAC) patients underwent elective PD or TP at Samsung Medical Center from 1995 to 2015. We reviewed cases that CTP was performed due to positive pancreatic margin. There were 21 cases that R0 were confirmed (cTP R0 group) and 4 cases that other positive margin were confirmed (cTP R1 group). In order to compare with cTP R0 group, 39 cases that R0 were confirmed after PD were selected through matching (PD R0 group). We compared postoperative outcomes between cTP R0 group and cTP R1 group, and between cTP R0 group and PD R0 group.

Results: In two comparative analysis, there were no significantly meaningful differences in clinical, operative, pathologic characteristics, and short-term postoperative outcomes classified Clavien-Dindo classification and hospitalization. The survival rate of cTP R0 group was higher than that of CTP R1 group, but the difference was not significant. The survival rate of cTP R0 group was lower than that of PD R0 group, which was significant.

Conclusions: In our center, we have not shown evidence that cTP for safe margin has significant benefit in survival. It cannot be concluded that CTP is unnecessary, and further research is needed in the future. 